\title{
Analytical Study on Inter-Cell Handover via Non-Concentric Circles in Wireless Heterogeneous Small Cell Networks
}

\author{
Hangyu $\mathrm{Gu}^{1}$, Shuangchun $\mathrm{Li}^{1}$, Vincent Havyarimana ${ }^{3}$, Dong Wang ${ }^{1}$, Zhu Xiao ${ }^{1,2}$ * \\ ${ }^{1}$ College of Computer Science and Electronic Engineering, Hunan University \\ 410082, Changsha, China \\ ${ }^{2}$ State Key Laboratory of Geo-Information Engineering, Xi'an, China \\ E-mail: \{guhangyu, lishuangchun, wangd, zhxiao\}@hnu.edu.cn, \\ ${ }^{3}$ Department of Applied Sciences, Ecole Normale Supérieure \\ 6983, Bujumbura, Burundi \\ havincent12@gmail.com \\ *Corresponding author: Zhu Xiao
}

Received October 27, 2017; revised December 1, 2017; accepted January 9, 2018; published May 31, 2018

\begin{abstract}
In this paper, we propose a novel inter-cell handover approach from a new perspective in dense Heterogeneous and Small Cell Networks (HetSNets). We first devise a cell selection mechanism to choose a proper candidate small cell for the UEs that tend to implement inter-small cell handover (ICH). By exploiting the property of a typical non-concentric circle, i.e., circle of Apollonius, we then propose a novel analytical method for modeling inter-cell handover regions and present mathematical derivation to prove that the inter-small cell handover issues fit the property of the circle of Apollonius. We design an inter-cell handover algorithm (ICHA) by means of our proposed handover model to dynamically configure hysteresis margin and properly implement handover decision in terms of UE's mobility. Simulation results demonstrate that the proposed ICHA yields lower call drop rate and radio link failure rate than the conventional methods and hence achieve high Handover Performance Indicator (HPI).
\end{abstract}

Keywords :Heterogeneous and Small cell Networks (HetSNets); Inter-Small cell Handover; Circle of Apollonius.

This work was partially supported by the National Natural Science Foundation of China (Grants Nos. 61301148, 61502162 and 61702175), the Fundamental Research Funds for the Central Universities of China, Fund of State Key Laboratory of Geoinformation Engineering (No. SKLGIE2016-M-4-2), Hunan Natural Science Foundation of China (No. 2018JJ2059), and Open Fund of State Key Laboratory of Integrated Services Networks (No. ISN17-14). 


\section{INTRODUCTION}

$\mathbf{W}_{\text {ith the anticipated high traffic, the implementation of Heterogeneous and Small cell }}$ Networks (HetSNets) are viewed as a promising approach to satisfy the ever-increasing demand for mobile users and business needs in the future 5th generation (5G) communication systems [1-3]. A large numbers of small cells including picocells and femtocells are deployed within the coverage of existing macrocells and thus forms multi-tier Heterogeneous and Small cell Networks (HetSNets), which provide an efficient way toward improving the transmission rate, enhancing the overall capacity and reducing the energy consumption of cellular communication networks $[4,5]$.Therefore, development of the HetSNets is one of the critical steps in the evolutionary direction of the future mobile cellular communication systems [6].

HetSNets are multi-tier network systems the network structure is more complex. Moreover, the variability of network factors, such as network types, technical standards and wireless connection in intra-tier and inter-tier scenarios, leads to various types of handover [7]. Therefore, designing a network structure with higher flexibility and lower complexity for the issues on mobility management and handover has become the focus of current research. The number of handovers is associated with the location of the small cell in HetSNets [8]. In particular, due to the dense deployment of small cells, the inter-small cell handover, namely user equipment (UE) moves out from one small cell and tends to handin an adjacent small cell, needs to be taken into consideration, since it is an essential way to avoid unnecessary handovers and thus reduce the radio link failure (RLF). Hence, a direct inter-small cell handover rather than a cross-tier small cell-macrocell-small cell is able to reduce the number of handovers, save network resources, and create more powerful network service capabilities of small cell tier. In addition, relying on an adequate and intensive deployment, small cells provide better link quality for their associated UEs, in the meantime the macrocell may shrink its coverage area by reducing its transmit power and hence the heterogeneous networks will become more spectral- and energy-efficient. As shown in [8], the authors studied the mobility and stability of the LTE-intensive small cell network after turning off the macrocells.

To fulfill the growth of the number of UE and address the connectivity between the UE and small cells, the existing works have made adequate contributions to the implementation of the small cell handover. In [9], the authors proposed a framework of 3C-oriented small cell networks with IA, in which caching and computing are exploited to simplify the network topology, improve the throughput, reduce the backhaul load, and guarantee the quality of experience of users. In [10], the article proposed a hierarchical cloud computing architecture to meet the increasing amount of traffic, quality requirements, and new mobile cloud computing demands in the emerging heterogeneous mobile network. In [11], power allocation was considered to improve the performance of cache-aided small-cell networks with limited backhaul. In [12], the authors provided a description of the fog radio access network architecture. In [13], the problems of cooperative interference mitigation and handover management in a heterogeneous cloud small cell network were examined. In [14], the authors introduced a logical architecture for network-slicing-based 5G systems, and present a scheme for managing mobility between different access networks, as well as a joint power and subchannel allocation scheme in spectrum-sharing two-tier systems based on network slicing. In [15], the cross- and co-tier Device-to-Device communications were modeled in two-tier macro-small cell networks. In [16], the authors developed the use of a selective C/U-plane split combined with a non-coherent cooperative multi-point joint transmission to deal with UEs mobility in a context of small cells operating at the millimeter wave. In [17], the secrecy spectrum efficiency and secrecy energy efficiency of a K-tier massive multiple-input 
multiple-output enabled heterogeneous cellular network were studied. In [18] the authors investigated the hierarchical clustering for dense small cells and devise non-cooperative game-theoretic scheme. In [19], a radio over fiber based model was proposed to solve the load balancing problem in ultra-dense network. In [20] the authors investigated the impact of mobility on user performance in the context of dense LTE-A networks. In [21], the open issues for mobility management support in the presence of femtocells and overviews the key aspects of mobility management in the LTE-A system were discussed. In [22], the authors introduced a new model for analyzing handover performance in heterogeneous networks. In [23], a state-dependent handover decision algorithm was proposed for the trade-of between the low handover failure rate and the high small cell utilization.

According to above literature review, the majority of previous works that study the handover in HetSNets are on the basis of concentric circle [20-23]. Specifically, the cell coverage and handover regions are modeled as concentric circles. Nevertheless, the concentric circle model for formulating handover has the insurmountable drawback. Suppose small cell $S$ and small cell $C$ are two adjacent small cells. When a UE is served by small cell $S$ and leaving out of small cell $S$, it can move towards its adjacent small cell $C$ or away from small cell $C$. We can reasonably presume that the received signal power from small cell $S$ is becoming weaker. But the received signal from small cell $C$ depends on the moving direction of UE. When the UE is moving towards small cell $C$, the received signal power from small cell $C$ is becoming stronger. On the other side, when UE is moving away from small cell $C$, the received signal power from small cell $C$ will decrease. Hence, the UE handover from small cell $S$ with different directions can lead to non-concentric circles for handover regions. We will present the detailed analysis in Sec. III

In this paper, we investigate inter-small cell handover in HetSNet from a new perspective. First, based on the analysis of channel conditions, we propose a cell selection mechanism such as to choose a proper candidate small cell for the UEs that tend to implement inter-small cell handover. By exploiting the circle of Apollonius, we then propose a novel analytical method for modeling inter-cell handover regions. Furthermore, we present mathematical derivation to prove that the inter-small cell handover issues fit the property of the circle of Apollonius. To achieve fast and efficient handover, we design an inter-cell handover algorithm (ICHA) by means of our proposed handover model to dynamically configure hysteresis margin and properly implement handover decision in terms of UE's mobility. Simulation results validate the effectiveness of our proposed handover model based on Apollonius circle. We consider Handover Performance Indicator (HPI) to evaluate the performance of our proposed method. The results demonstrate that the proposed method yields lower call drop rate and radio link failure rate than the conventional methods during inter-small cell handovers.

The rest of the paper is organized as follows. In Section II, we present the cell selection mechanism. In Section III, we provide analytical study on handover regions based on non-concentric circles. The Inter-Small Cell Handover algorithm is presented in Section IV. The effectiveness of ICHA are demonstrated by simulation results in Section V. Finally, we conclude the paper in Section VI.

\section{CELL SELECTION MECHANISM}

In the dense small cell deployment scenario, when a UE attempts to move cross its associated small cell and handover to an adjacent small cell, there will be likely multiple target small cells. To avoid the handover confusion [24], we design a cell selection mechanism with purpose of finding out a unique candidate small cell for the UEs that tend to implement inter-cell handover. 
A proper target small cell should be selected with better channel condition to maintain good connectivity and link quality for UEs that trigger the handover process. Assume that the system spectrum in multi-tier HetSNets available for small cell tier consists of $N$ bands denoted by $\Omega=\{1,2, \ldots, N\}$. $C_{n}$ denotes the set of small cells using band $n(n \in \Omega)$. Let $U_{n}$ denote the total number of UEs in the small cell tier. We consider Orthogonal Frequency Division Multiplexing (OFDM) based heterogeneous macrocell-small cell networks. Assume $R_{n}$ resource blocks (RBs) are used in each time slot. $R_{D L}^{C}\left(R_{D L}^{C} \subseteq R_{n}\right)$ denotes the number of the RBs in the downlink of small cell $C$. The reference signal receiving power (RSRP) of each $\mathrm{RB} r$ in $\mathrm{R}_{\mathrm{DL}}^{\mathrm{C}}$ can be expressed as $\mathrm{P}_{\mathrm{r}, \mathrm{RS}}^{\mathrm{C}} \cdot h_{r}^{C U}$ is the channel gain of $r$ and $\mathrm{UE} U$. The minimum RSRP for maintaining the connection between a small cell and a UE is $R S R P_{t h}$. It is assumed that $\mathrm{UE} U$ is served by small cell $S$ and is approaching small cell $C\left(C \in \cup_{n=1}^{N} C_{n}, C \neq S\right)$.

Considering hype dense small cell deployments and fast-changing wireless network, maintaining the connectivity of the terminal to the small cell is very important. In the deployment of a small cell, the high RSRP generally denotes the high channel gain. However, a high RSRP is inclined to the small cell with a high RSRP in the deployment of the small cell. Handover to the small cell with a high RSRP is not meant to improve the channel gain or SINR performance between the UE and small cell [22]. Therefore, from the perspective of channel gain, the small cell selecting mechanism is derived as follows

According to the definition of RSRP, UE $U$ receiving RSRP (W) from small cell $C$ was formulated as:

$$
\operatorname{RSRP}^{C U}=\frac{\sum_{r \in R_{D L}^{C}} P_{r, R S}^{C} \cdot h_{r}^{C U}}{R_{D L}^{C}}
$$

We replace the average $P_{R S}^{C}$ with $P_{r, R S}^{C}$ in the downlink of small cell $C$ :

$$
\Rightarrow \operatorname{RSRP}^{C U}=P_{R S}^{C} \cdot \frac{\sum_{r \in R_{D L}^{C}} h_{r}^{C U}}{R_{D L}^{C}}
$$

where $\frac{\sum_{r \in R_{D L}^{C}} h_{r}^{C U}}{R_{D L}^{C}}$ indicates that the average of the sum of the channel gains of all RBs in the downlink small cell $C$ is linearly averaged. The average channel gain $h^{C U}$ between small cell $C$ and UE $U$ can be formulated as:

$$
h^{C U}=\frac{\sum_{r \in R_{D L}^{C}} h_{r}^{C U}}{R_{D L}^{C}}
$$

Therefore, (1) can be simplified as:

$$
\operatorname{RSRP}^{C U}=P_{R S}^{C} \cdot h^{C U}
$$

Let $P_{\max }^{U}$ and $P_{\max }^{C}$ denote the maximum transmit powers of UE $U$ and small cell $C$, respectively. The channel gain $h_{\text {min }}^{C U}$ beween small cell $C$ and UE $U$ can be expressed as follows:

$$
h_{\min }^{C U}=\frac{R S R P_{\text {th }}}{\min \left(P_{\max }^{U}, P_{\max }^{C}\right)}
$$

Therefore, the connectivity is maintained by satisfying the channel gain between the UE and the associated small cell:

$$
h^{C U} \geq h_{\min }^{C U}
$$

According to (4), (5) and (6), we have:

$$
\frac{\mathrm{RSRP}^{C U}}{P_{R S}^{C U}} \geq \frac{R S R P_{t h}}{\min \left(P_{\text {max }}^{U}, P_{\text {max }}^{C}\right)}
$$

By converting formula (7) into $\mathrm{dB}$ form, a decision criterion is used to determine whether the small cell channel conditions are satisfactory. The criterion can be used to select out the small 
cells with better channel conditions for the UE and form the candidate small cell set when it performs the handover measurement $\phi$ :

$$
\begin{gathered}
\mathrm{Mi}=\min \left(P_{\max }^{U}, P_{\max }^{C}\right) \\
\phi=\left\{\mathrm{C} \mid \operatorname{RSRP}^{C U} \geq R S R P_{t h}+P_{R S}^{C}-\mathrm{Mi}, \mathrm{C} \in \Psi\right\}
\end{gathered}
$$

\section{Inter-cell handover based on circle of Apollonius}

We propose a novel Inter-Small cell handover model in this section. We first show that the traditional analytical model is not suitable for the handover scenario. Then we build the novel model by employing the property of the circle of Apollonius. The Inter-Small cell handover model based on the Apollonius circle is shown in Fig. 1.

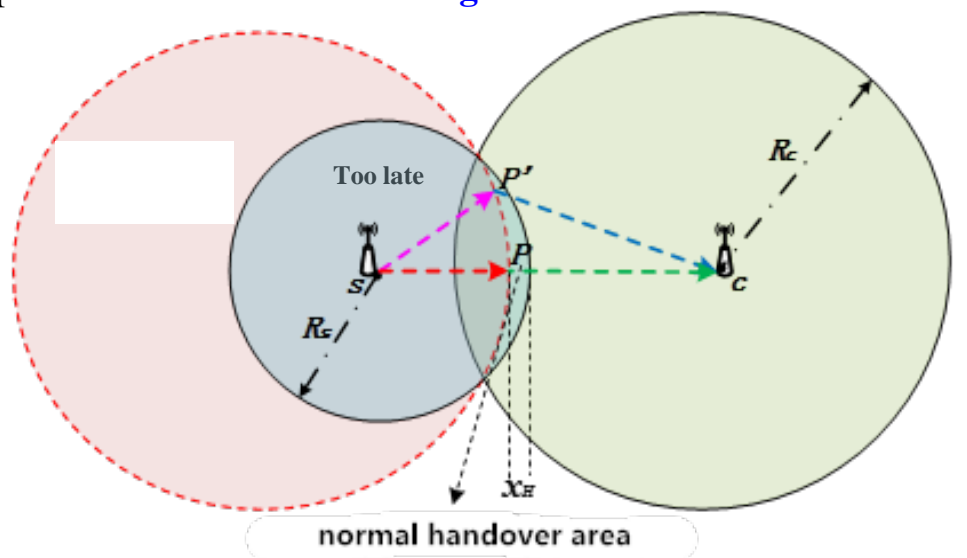

Fig. 1. Inter-Small cell handover

\section{A. Traditional handover model}

In the current literatures, for instance in [20-23], the handover models are based on concentric circles. This may be inappropriate to formulate the handover regions. Consider a scenario where a UE intends to handover from its serving small cell $S$ to small cell $C$. The average received signal powers from small cell $S$ and small cell $C, P_{r, S}$ and $P_{r, C}$, can be expressed by

$$
\begin{aligned}
P_{r, S} & =P_{S} d_{U, S}^{-\alpha} \chi_{S} h^{S U} \\
P_{r, C} & =P_{C} \mathrm{~d}_{U, C}^{-\beta} \chi_{C} h^{C U}
\end{aligned}
$$

$P_{S}$ and $P_{C}$ are the transmit powers of small cell $S$ and small cell $C . d_{U, S}$ and $d_{U, C}$ denote the distance from the UE to small cell $S$ and small cell C. $\alpha$ and $\beta$ are the path loss exponents of the small cell $S$ and the small cell $C$. $\chi_{S}$ and $\chi_{C}$ denote the propagation shadowing from UE to small cell $S$ and small cell $C . h^{S U}$ and $h^{C U}$ are the average channel gains from small cell $S$ and $C$ to UE. $\operatorname{SINR}_{S}$ and $S I N R_{C}$ are Signal-to-Interferenceplus-Noise-Ratios (SINR) that the UE receives from the small cell $S$ and $C$.

$$
\begin{aligned}
& \operatorname{SINR}_{S}=\frac{P_{r, S}}{P_{r, C}+\sigma^{2}}=\frac{P_{S} d_{U, S}^{-\alpha} \chi_{S} h^{S U}}{P_{C} d_{U, C}^{-\beta} \chi_{C} h^{C U}} \\
& \operatorname{SINR}_{C}=\frac{P_{r, C}}{P_{r, S}+\sigma^{2}}=\frac{P_{C} d_{U, C}^{-\beta} \chi_{C} h^{C U}}{P_{S} d_{U, S}^{-\alpha} \chi_{S} h^{S U}}
\end{aligned}
$$

According to the 3GPP standard [26], when the receiving SINR from the target cell or serving cell is less than $-6 \mathrm{~dB}$, the handover is too early or too late. 


$$
\begin{array}{r}
10 \log \frac{P_{S} d_{U, S}^{-\alpha} \chi_{S} h^{S U}}{P_{C} d_{U, C}^{-\beta} \chi_{C} h^{C U}} \leq-6 \\
10 \log \frac{P_{C} d_{u, c}^{-\beta} \chi_{c} h^{C U}}{P_{S} d_{U, S}^{-\alpha} \chi_{S} h^{S U}} \leq-6
\end{array}
$$

$d_{E S}$ and $d_{E C}$ are the distances from the UE to the small cell $S$ and the small cell $C$ in the too early handover case. Similarly, $d_{L S}$ and $d_{L C}$ are the distances from the UE to the small cell $S$ and the small cell $C$ in the too late handover case.

$$
\begin{aligned}
& \lambda_{E}=\frac{d_{E C}}{d_{E S}} \\
& \lambda_{L}=\frac{d_{L C}}{d_{L S}}
\end{aligned}
$$

Based on Eqs. (14)(15)(16)(17), we obtain

$$
\begin{aligned}
& \lambda_{E} \leq\left(\frac{P_{C} \chi_{C} d_{E, C}^{\alpha-\beta} h^{C U}}{4 P_{S} \chi_{S} h^{S U}}\right)^{\frac{1}{\alpha}} \\
& \lambda_{L} \geq\left(\frac{4 P_{C} \chi_{C} d_{L, C}^{\alpha-\beta} h^{C U}}{P_{S} \chi_{S} h^{S U}}\right)^{\frac{1}{\alpha}}
\end{aligned}
$$

We define $c^{*}$ and $s^{*}$ as follow:

$$
\begin{aligned}
& c^{*}=\max \left\{\left(\frac{P_{C} \chi_{C} d_{E, C}^{\alpha-\beta} h^{C U}}{4 P_{S} \chi_{S} h^{S U}}\right)^{\frac{1}{\alpha}}\right\} \\
& s^{*}=\min \left\{\left(\frac{4 P_{C} \chi_{C} d_{L, C}^{\alpha-\beta} h^{C U}}{P_{S} \chi_{S} h^{S U}}\right)^{\frac{1}{\alpha}}\right\}
\end{aligned}
$$

Apollonius circle can be defined as the set of points in a plane that have a specified ratio of distances to two fixed points. In this model small cell $S$ and $C$ are fixed points. Based on Eq. (20)(21), with different values of $\lambda_{E}, \lambda_{L}$, the trajectories will be a set of Apollonius circles. $c^{*}$ and $s^{*}$ denote the boundaries of the early and late handover region, respectively. All possible values of $\lambda_{E}, \lambda_{L}$ are included in the circles.

\section{B. Normal handover based on circle of Apollonius}

$d_{H S}$ and $d_{H C}$ are the distances from the UE to the small cell $S$ and the small cell $C$ in the normal handover case. $H$ is the hysteresis margin

$$
\lambda_{H}=\frac{d_{H C}}{d_{H S}}
$$

The normal handover condition can be formulated from the Reference Signal Receiving Power (RSRP) perspective

$$
\operatorname{RSRP}(M) \geq \operatorname{RSRP}(S)+H
$$

Change it to another modality, we can obtain

$$
\frac{p_{r, c}}{p_{r, s} H} \geq 1
$$

Based on Eqs. (10) (11), $P_{r, S}=P_{S} d_{U, S}^{-\alpha} \chi_{S} h^{S U}, P_{r, C}=P_{C} \mathrm{~d}_{U, C}^{-\beta} \chi_{C} h^{C U}$ we then have

$$
\frac{P_{C} \chi_{C} d_{H, C}^{-\beta} h^{C U}}{P_{S} \chi_{S} d_{H, S}^{-\alpha} h^{S U} H} \geq 1
$$


Based on Eqs.(12), (13) and (22), we obtain $\lambda_{H}$ which can be given as follow:

$$
\lambda_{H} \leq\left(\frac{P_{C} \chi_{C} d_{H, C}^{-\beta} h^{C U}}{P_{S} \chi_{S} d_{H, S}^{-\alpha} h^{S U} H}\right)^{\frac{1}{\alpha}}
$$

Assuming that the total time spent by the UE to complete the handover at the time of arrival of the boundary of small cell $S$ is $T T T+T_{0}$, the distance traveled at that time period is:

$x_{H}$ can be approximated as:

$$
x_{H}=\mathrm{v} \cdot\left(T T T+T_{0}\right)
$$

$$
\begin{gathered}
x_{H} \approx R_{S}-d_{U, S} \\
\Rightarrow d_{U, S}=R_{S}-v \cdot\left(T T T+T_{0}\right)
\end{gathered}
$$

According to the feature of the Apollonius circle:

$$
\begin{gathered}
\frac{d_{U, S}}{d_{U, C}}=\frac{R_{S}}{R_{C}} \\
\Rightarrow d_{U, C}=\left[R_{S}-v \cdot\left(T T T+T_{0}\right)\right] \cdot \frac{R_{C}}{R_{S}}
\end{gathered}
$$

The formulas (29) and (31) are substituted into formula (25) to obtain the hysteresis automatic configuration formula based on the terminal speed of Inter-Small cell handover:

$$
\mathrm{H}(U)=\frac{P_{C} \cdot h^{C U}}{P_{S} \cdot h^{S U}} \cdot\left(\frac{R_{S}}{R_{C}}\right)^{\beta} \cdot\left[R_{S}-v \cdot\left(T T T+T_{0}\right)\right]^{\alpha-\beta}
$$

where $R_{S}$ and $R_{C}$ is the radius of service small cell $S$ and candidate small cell $C$, respectively. In the formula, terminal speed $v$ is the only variable, and the other parameters are constants. Thus, the derived formula of hysteresis margin can be dynamically configured according to the UE's speed.

A normal handover is performed when the UE moves to a certain location in this area. At this time, the ratio of the distance from the UE to these two small cells is described by the Apollonius circle. Hence, the boundary of the Apollonius circle and its service small cell boundary will be perceived as a normal handover (as shown in Fig. 1 in the red dotted line). The proposed model is an improvement compared with the traditional concentric circle model. Although we focus on investigating the inter-cell handover in co-channel scenario, it is also applicable for the scenario when dedicated channel is used in small cells.

\section{Inter-cell handover algorithm (ICHA)}

We propose the inter-cell handover algorithm (ICHA) in this section. The procedure of proposed ICHA is presented in Fig. 2:

Step 1: The algorithm is used to measure each small cell in the $\Psi$ set;

Step 2: By using this measurement information, the proposed algorithm first performs the small cell selection mechanism to select a candidate small cell that matches the proposed criteria; 
Step 3: If a candidate small cell meets its requirement, the proposed algorithm implements the dynamic allocation mechanism based on the UE speed and configures the dynamic delay margin according to the UE speed.

Step 4: If a small cell in these candidate small cell set satisfies the inter-cell handover condition, then the UE starts to implement the handover process to the small cell. After a certain period of time to study the cell, the algorithm repeats step (2)-step (4);

Step 5: If a candidate small cell does not exit, the UE starts to re-search the small cells in the area. Moreover, the algorithm starts the cycle again and returns to step (2);

Step 6: If the candidate small cell cannot satisfy the handover entry condition, then the UE does not perform a handover and continues to stay in its associated small cell. After a certain period of time to re-search for a cell, the algorithm begins again and returns to step (2).

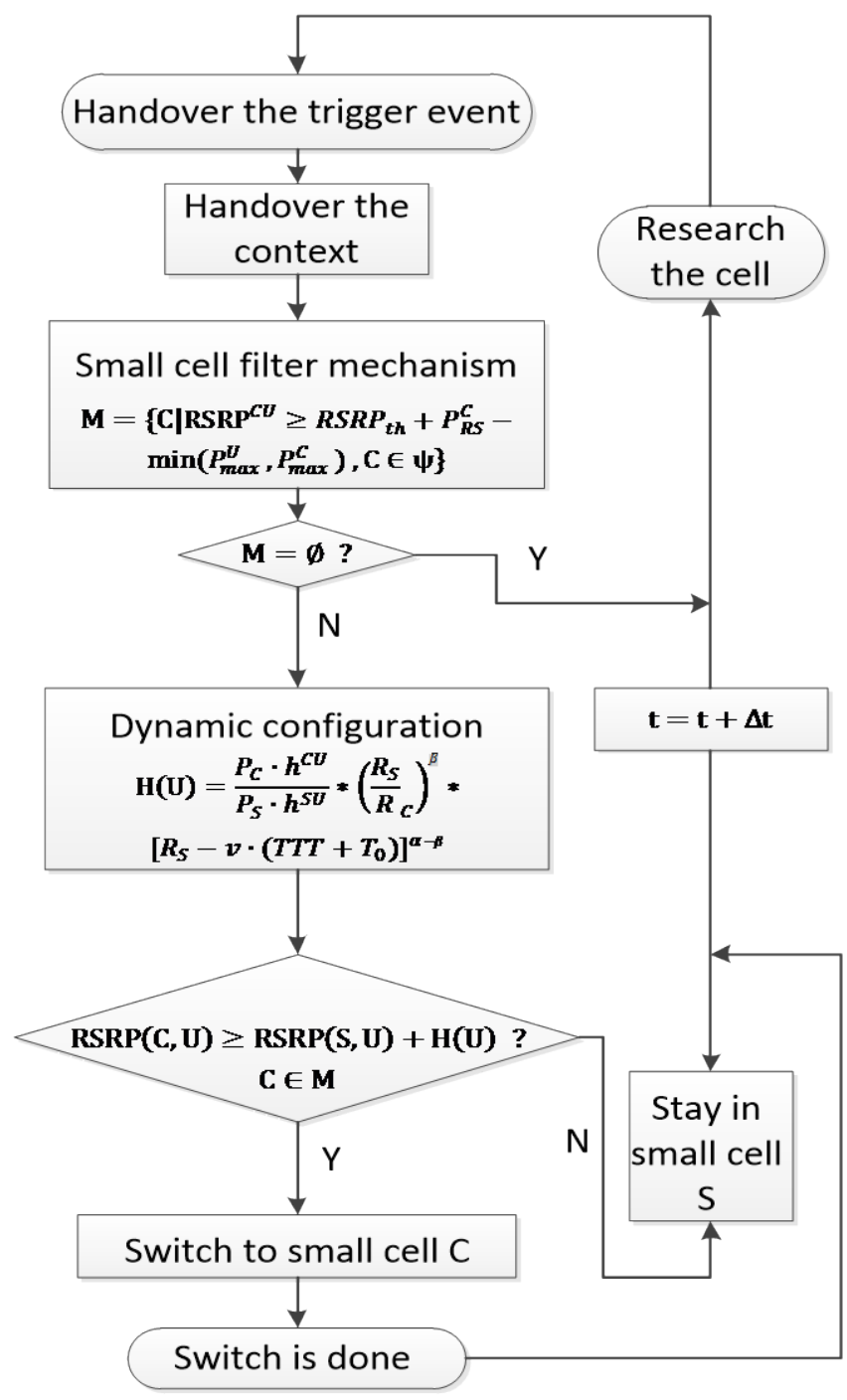

Fig. 2. Flow diagram of the ICHA

\section{SIMULATIONS AND PERFORMANCE EVALUATION}

In this section, we evaluate the system performance of the proposed ICHA and compare it with the traditional algorithm [22-23] in the outbound handover. Based on [27], we use RSRP with 
hysteresis for UE to determine whether it would trigger handover, and in the meantime, to avoid too early or late handover by considering the UE's velocity. We also assume that the UEs move with a random direction, according to the wrap around model in [27]. The key parameters are listed in Table 1.

Table 1. Simulation parameters

\begin{tabular}{|c|c|}
\hline Carry frequency/system bandwidth & $2 \mathrm{GHz} / 10 \mathrm{MHz}$ \\
\hline Tx power of macrocell / small cell & $46 \mathrm{dBm} / 20 \mathrm{dBm}$ \\
\hline Macrocell/small cell radius & $500 \mathrm{~m} / 25 \mathrm{~m}$ \\
\hline $\begin{array}{c}\text { Log-normal shadowing macrocell } \\
\text { standard deviation } 8 \mathrm{~dB}\end{array}$ & $8 \mathrm{~dB} / 4 \mathrm{~dB}$ \\
Small cell standard deviation & $500 \mathrm{~m}$ \\
\hline The distance between base stations & $15.34+37.6 * \log ^{10(\mathrm{~d}(\mathrm{~m}))}+\mathrm{WL} 10(\mathrm{~m}) \leq \mathrm{d} \leq 20(\mathrm{~m})$ \\
\hline Macrocell path loss [28] & $15.3+37.6 * \log ^{10(\mathrm{~d}(\mathrm{~m}))} \quad \mathrm{d} \geq 20(\mathrm{~m})$ \\
\hline Small cell pass loss & $38.46+20 * \log ^{10(\mathrm{~d}(\mathrm{~m}))}+0.7 \mathrm{~d}_{\min } \quad 10(\mathrm{~m}) \leq \mathrm{d} \leq 20(\mathrm{~m})$ \\
& $38.46+20 * \log ^{10(\mathrm{~d}(\mathrm{~m}))}+0.7 \mathrm{~d}_{\min }+\mathrm{WL} \quad \mathrm{d} \geq 20(\mathrm{~m})$ \\
\hline Terminal quantity/velocity/dir-ection & $100 / 1,1.5,2, \ldots, 10 \mathrm{~m} / \mathrm{s} / \mathrm{direct} \mathrm{movement}$ \\
\hline Hysteresis margin $/ T T T / T_{0}$ & Dynamic configuration $/ 100 \mathrm{~ms} / 150 \mathrm{~ms}$ \\
\hline
\end{tabular}

\section{A. Handover hysteresis margin}

In this simulation, the distance of the source small cell with a radius of $50 \mathrm{~m}$ from the target small cell with a radius of $100 \mathrm{~m}$ is $130 \mathrm{~m}$, where they have an overlapping area of up to $20 \mathrm{~m}$.

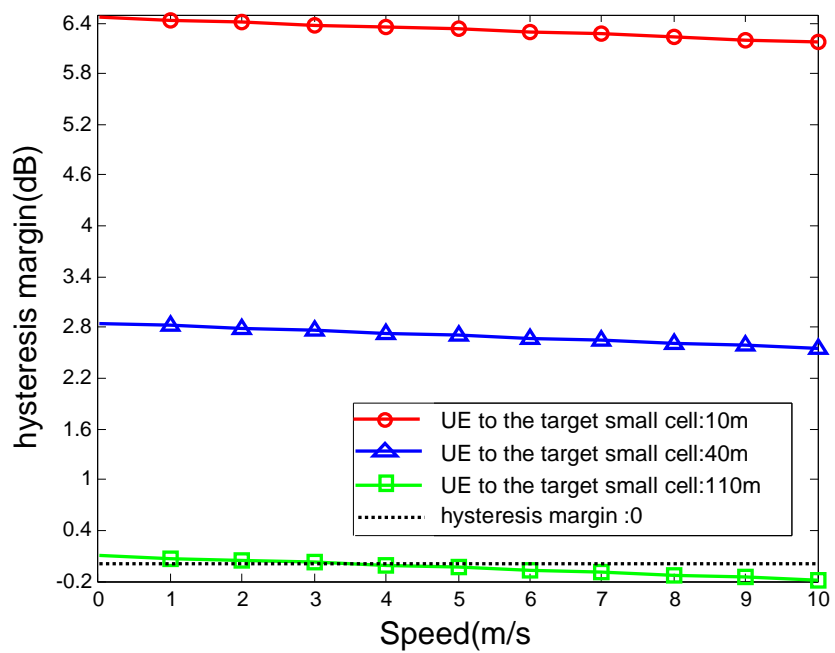

Fig. 3. Hysteresis margin vs. UE’s velocity

Fig. 3 shows the trend of the hysteresis margin at each terminal speed; the trend illustrates that the proposed algorithm is very sensitive to the change of speed regardless of the location of the UE. Hence, a small cell promotes or prevents its implementation of the handover by reducing or increasing the hysteresis margin. We select the three scenarios according to the distance from the UE to the source small cell: the UE is located in the overlay of source small cell $(10 \mathrm{~m})$, the overlapping area $(40 \mathrm{~m})$ of the two small cells or the coverage area of the target small cell $(110 \mathrm{~m})$. The hysteresis margins configured in the three scenarios decrease in turn and decrease with the increase of the terminal speed; this feature is helpful to meet the handover requirements of the UE at each speed. 


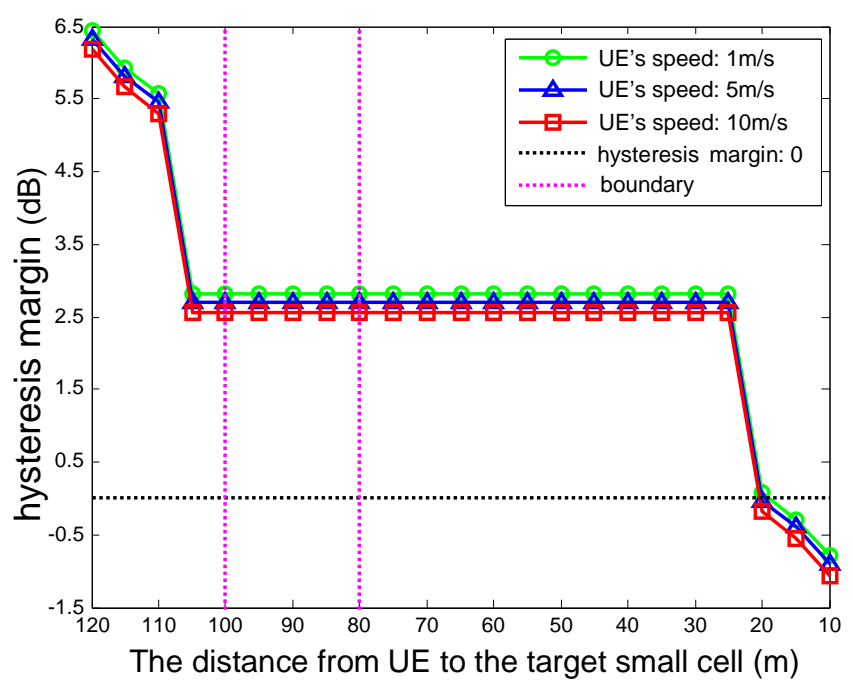

Fig. 4. Hysteresis margin vs. the distance from the UE to the target small cell

Fig. 4 shows the trend of the hysteresis margin at each distance. When the UE is close to the source small cell, a high hysteresis margin value is required, regardless of the UE's speed, to reduce its impact from the nearby small cell and frequent performing of handovers. The hysteresis margin decreases and the handover to the adjacent small cell becomes easier for the UE when the UE gradually moves away from the source small cell. We consider the following three conditions: low speed $(1 \mathrm{~m} / \mathrm{s})$, medium speed $(5 \mathrm{~m} / \mathrm{s})$ and high speed $(10 \mathrm{~m} / \mathrm{s})$. The overall situation of the hysteresis margin in the three cases decreases as the UE approaches the target small cell; thus, the proposed algorithm is in line with expectations.

It is noteworthy that the magenta vertical lines in the figure represent the overlapping area boundaries $(100 \mathrm{~m}, 80 \mathrm{~m})$ of the two small cells. In the left side of the magenta vertical line, the declining trend of the hysteresis margin is very obvious. At this point, once the UE enters the handover area, the proposed algorithm for its configuration significantly reduces the hysteresis margin to assist in its handover. In addition, within the two magenta vertical lines, the hysteresis margin remains unchanged because the UE is executing or has completed the handover process. This is consistent with the assumption that the UE had completed the handover before leaving of the overlapping area. When the UE is close to the target small cell, the hysteresis margin decreases again; this decrease is caused by the path loss model (the distance between the UE and the small cell is different, and the path loss model expression is also different).

Fig. 4 and Fig. 5 illustrate that the proposed algorithm can be a dynamically adjusted hysteresis margin based on the speed of UE and the distance from the UE to the target small cell, which conforms to the standard that the hysteresis margin is automatically determined in the heterogeneous small cell network.

\section{B. Performance evaluation}

This part examines the handover performance of the proposed algorithm. In this simulation, the UE is divided into two types: low-speed UE, with a speed of $1 \mathrm{~m} / \mathrm{s}$, and high-speed UE, with a speed of $10 \mathrm{~m} / \mathrm{s}$. We compare this algorithm with the traditional centralized control scheme (fixed hysteresis margin). 


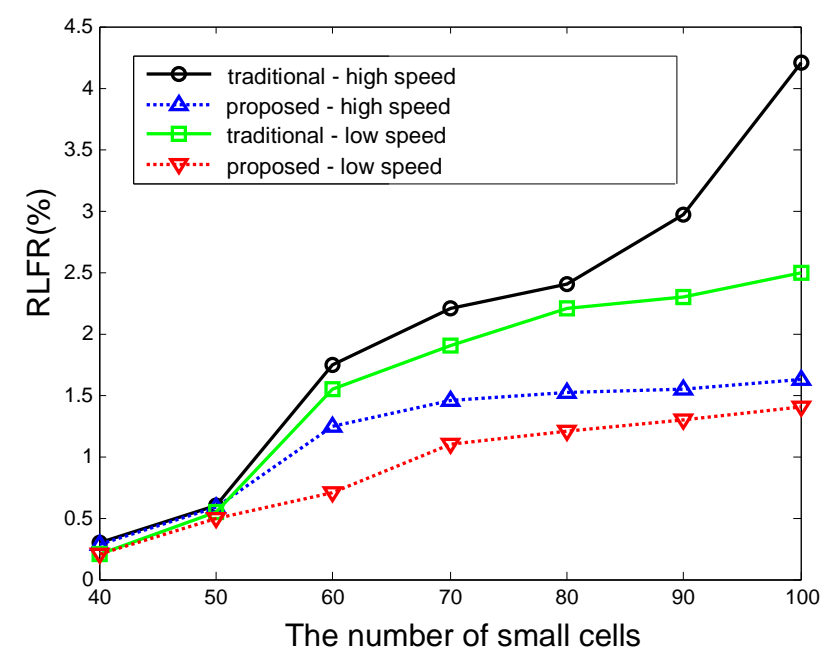

Fig. 5. Average RLFR - the proposed scheme vs. the traditional scheme

Fig. 5 shows a comparison of the average Radio Link Failure Rate (RLFR) of the proposed algorithm with that of the traditional handover scheme. With the increase in the number of small cells, the interference and abnormal handover in the system network is also increased, resulting in Radio Link Failure (RLF). The traditional handover scheme does not remove the small cell with poor channel conditions for the UE; as a result, RLF is likely to occur when the terminal device is connected to it. At the same time, the fixed hysteresis margin causes some terminals to handover too early or to handover too late and may also cause RLF. The proposed algorithm utilizes the small cell screening mechanism to select some small cells with good channel conditions for the terminal devices to avoid the reconnection problem caused by the poor signal quality and dynamically configures the hysteresis margin according to its speed to avoid improper setting of the hysteresis margin caused by the early and late handover. The combination of two stages can reduce the RLFR.

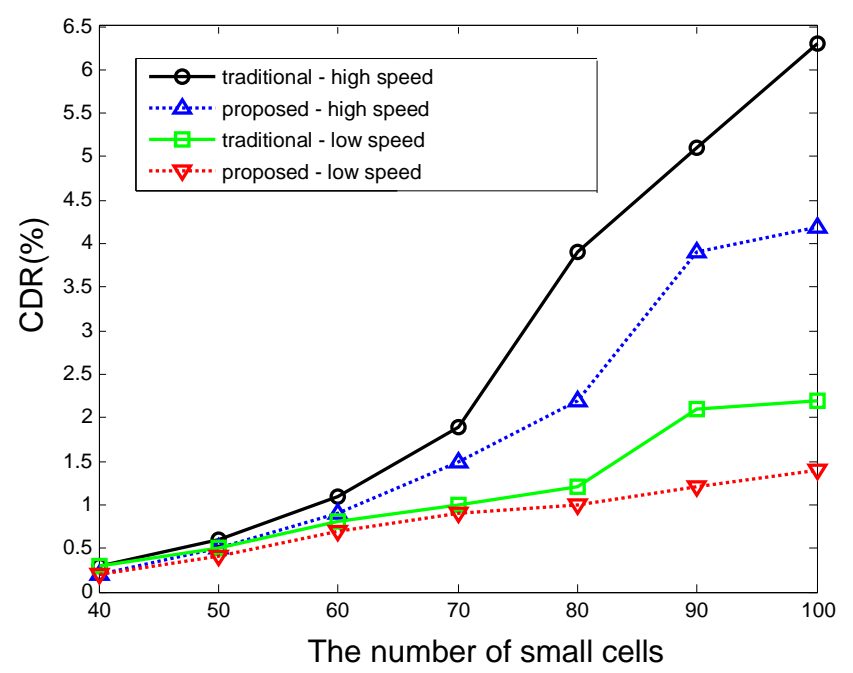

Fig. 6. Average CDR - the proposed scheme vs. the traditional scheme

Fig. 6 shows a comparison of the average Call Drop Ratio (CDR) of the proposed algorithm with that of the traditional handover scheme. With the increase in the number of 
small cells, the number of terminal devices is also increased. A small cell does not have sufficient resources to serve such a large number of terminal devices. In addition, the amount of interference is increased, which seriously damages some channels of the small cell and in turn increases the CDR. The handover scheme of the traditional direct measurement of the RSRP does not eliminate the small cell with poor channel conditions for the terminal devices, which makes it possible for the UE to connect to this small cell and lead to a Call Drop (CD). The uniform hysteresis margin causes the low-speed terminal devices to perform the handover too early or the high-speed terminal to perform the handover too late, even causing the RLF to suffer a CD. The proposed algorithm first uses the small cell screening mechanism to select the small cell with better channel conditions for the UE, and then use the hysteresis margin dynamic configuration mechanism to configure the appropriate hysteresis margin for the terminal devices. The combination of two stages can reduce the CDR.

To further study the handover performance of the proposed algorithm, the HPI (Handover Performance Indicator) is proposed based on CDR and RLFR. The definition is as follows:

$$
H P I=\frac{w_{C D} \cdot C D R+w_{R L F} \cdot R L F R}{w_{C D}+w_{R L F}}
$$

where $w_{C D}$ and $w_{R L F}$ represent the weights of CD and RLF, respectively. Since RLF sometimes results in CD, we specify $w_{R L F}=1, \quad w_{C D}=0.5$. Obviously, a lower HPI leads to better performance.

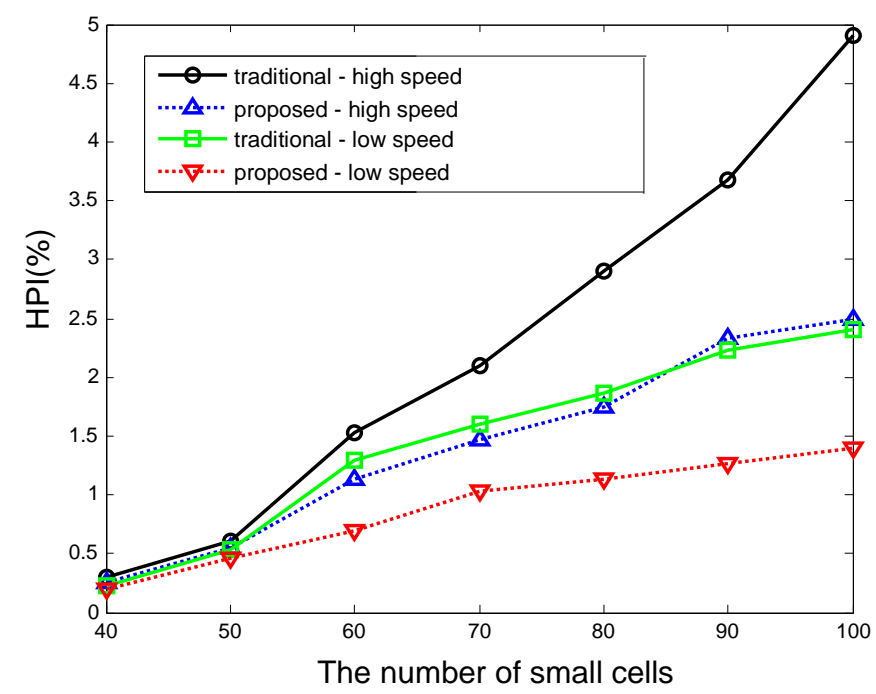

Fig. 7. Average HPI - the proposed scheme vs. the traditional scheme

Fig. 7 shows the HPI comparison of the proposed algorithm and the traditional scheme. In both cases, the proposed HPI is lower than that of the traditional handover scheme. CD and RLF are concerned with the small cell deployment density, interference, signal quality, the number of UEs and abnormal handover. The proposed algorithm can optimize the UE's wireless environment in two stages to further reduce abnormal handover phenomenon. This also reflects that neighborhood optimization and the use of dynamic handover parameters are the appropriate means to improve handover performance in heterogeneous small cell networks. In terms of CD and RLF, the proposed small cell handover decision algorithm outperforms the traditional approaches. 


\section{Conclusions}

In this paper, we propose a novel model to formulate inter-cell handover in dense heterogeneous macrocell and small cell networks. By utilizing the property of Apollonius circles, we prove that the traditional concentric circles are inappropriate to model the handover regions. We then propose a novel analytical method for modeling inter-small cell handover based on Apollonius circles. Furthermore, we design an inter-cell handover algorithm (ICHA) based on our proposed handover model to dynamically adjust hysteresis margin and properly implement handover decision in terms of UE's mobility. Simulation results validate the effectiveness of our proposed handover model based on Apollonius circle in terms of HPI. The results demonstrate that the proposed method is able to achieve lower call drop rate and radio link failure rate than the traditional methods during inter-small cell handovers.

\section{Acknowledgements}

This work was partially supported by the National Natural Science Foundation of China (Grants Nos. 61301148, 61502162 and 61702175), the Fundamental Research Funds for the Central Universities of China, Fund of State Key Laboratory of Geoinformation Engineering (No. SKLGIE2016-M-4-2), Hunan Natural Science Foundation of China (No. 2018JJ2059), and Open Fund of State Key Laboratory of Integrated Services Networks (No. ISN17-14).

\section{References}

[1] D. López-Pérez, M. Ding, H. Claussen, and AH. Jafari, “Towards 1 Gbps/UE in cellular systems: Understanding ultra-dense small cell deployments,” IEEE Communications Surveys and Tutorials, vol. 17, no. 4, pp. 2078 - 2101, June, 2015. Article (CrossRef Link)

[2] M. Ding, D. Lopez-Perez, G. Mao, and P. Wang, "Will the Area Spectral Efficiency Monotonically Grow as Small Cells Go Dense? ” in Proc. of GLOBECOM 2015: Global Communications Conference, pp. 1-7, December 6-10, 2015. Article (CrossRef Link)

[3] Z. Xiao, H. Liu, V. Havyarimana, T. Li and D. Wang, "Analytical Study on Multi-Tier 5G Heterogeneous Small Cell Networks: Coverage Performance and Energy Efficiency,” Sensors, vol. 16, no. 11, 17 pages, 2016. Article (CrossRef Link)

[4] S. Samarakoon, M. Bennis, W. Saad, M. Debbah, and M. Latva-Aho, "Ultra dense small cell networks: turning density into energy efficiency," IEEE Journal on Selected Areas in Communications, vol. 34, no. 5, pp. 1267-1280, March, 2016. Article (CrossRef Link)

[5] A. D. L. Fuente, R. P. Leal, and A. G. Armada, "New technologies and trends for next generation mobile broadcasting services,” IEEE Communications Magazine, vol. 54, no. 11, pp. 217-223, October, 2016. Article (CrossRef Link)

[6] Z. Xiao, S. Li, X. Chen, D. Wang, W. Chen “A Load-Balancing Energy Consumption Minimization Scheme in 5G Heterogeneous Small Cell Wireless Networks Under Coverage Probability Analysis,” International Journal of Pattern Recognition and Artificial Intelligence, Vol .31, No.7, 21 pages, 2017. Article (CrossRef Link)

[7] T. Yamamoto, and S. Konishi, "Impact of small cell deployments on mobility performance in LTE-Advanced systems,” in Proc. of PIMRC Workshops 2013: International Symposium on Personal, Indoor and Mobile Radio Communications, pp.189-193, September 8-9, 2013. Article (CrossRef Link)

[8] X. Gelabert, G. Zhou, and P. Legg, "Mobility performance and suitability of macro cell power-off in LTE dense small cell HetNets,” in Proc. of CAMAD 2013: International Workshop on Computer Aided Modeling and Design of Communication Links and Networks, pp.99-103, September 25-27, 2013. Article (CrossRef Link) 
[9] N. Zhao, X. Liu, F. R. Yu, M. Li, and V. C. M. Leung, “Communications, caching, and computing oriented small cell networks with interference alignment,” IEEE Communications Magazine, vol. 54, no. 9, pp.29-35, September, 2016. Article (CrossRef Link)

[10] M. Jo, T. Maksymyuk, B. Strykhalyuk, and C. H. Cho, "Device-to-device-based heterogeneous radio access network architecture for mobile cloud computing," IEEE Wireless Communications, vol. 22, no. 3, pp.50-58, July, 2015. Article (CrossRef Link)

[11] F. Cheng, Y. Yu, Z. Zhao, N. Zhao, Y. Chen, and H. Lin, “ Power allocation for cache-aided small-cell networks with limited backhaul,” IEEE Access, vol. 5, pp.1272-1283, January, 2017. Article (CrossRef Link)

[12] H. Zhang, Y. Qiu, X. Chu, K. Long, and V. C. M. Leung, “ Fog radio access networks: mobility management, interference mitigation and resource optimization”.

[13] H. Zhang, N. Liu, X. Chu, K. Long, A. H. Aghvami, and V. C. M. Leung, “ Network slicing based $5 \mathrm{~g}$ and future mobile networks: mobility, resource management, and challenges,” IEEE Communications Magazine, vol. 55, no. 8, pp.138-145, August, 2017. Article (CrossRef Link)

[14] H. Zhang, C. Jiang, and J. Cheng, "Cooperative interference mitigation and handover management for heterogeneous cloud small cell networks,” IEEE Wireless Communications, vol. 22, no. 3, pp.92-99, July, 2015. Article (CrossRef Link)

[15] T. Li, Z. Xiao, H. M. Georges, Z. Luo, and D. Wang, "Performance analysis of co- and cross-tier device-to-device communication underlaying macro-small cell wireless networks,” KSII Transactions on Internet and Information Systems, vol. 10, no .4, pp. 1481-1500, April, 2016. Article (CrossRef Link)

[16] M. Joud, M. Garcia-Lozano and S. Ruiz, "On the mobility of moderate speed users in ultra dense small cell deployments with mmw," in Proc. of VTC Spring 2015: Vehicular Technology Conference, pp. 1-5, May 11-14, 2015. Article (CrossRef Link)

[17] Z. Zhong, J. Peng, K. Huang, L. Xia and X. Qi, "Secrecy spectrum and secrecy energy efficiency in massive mimo enabled hetnets,” KSII Transactions on Internet and Information Systems, vol. 11, no. 2, pp.628-649, February, 2017. Article (CrossRef Link)

[18] Z. Xiao, J. Yu, T. Li, Z. Xiang, D. Wang, and W. Chen, "Resource allocation via hierarchical clustering in dense small cell networks: A correlated equilibrium approach,” in Proc. of Personal, Indoor, and Mobile Radio Communications, September 4-8 2016. Article (CrossRef Link)

[19] H. Xu, Z. He and X. Zhou, "Load balancing algorithm of ultra-dense networks,” KSII Transactions on Internet and Information Systems, vol. 9, no.7, pp. 2454-2467, July, 2015. Article (CrossRef Link)

[20] B. Baynat, R. M. Indre, N. Nya and P. Olivier, "Impact of Mobility in Dense LTE-A Networks with Small Cells,” in Proc. of VTC Spring 2015: Vehicular Technology Conference, pp.1-5, May 11-14, 2015. Article (CrossRef Link)

[21] D. Xenakis, N. Passas, L. Merakos and C. Verikoukis, "Mobility management for femtocells in lte-advanced: key aspects and survey of handover decision algorithms," IEEE Communications Surveys and Tutorials, vol. 16, no. 1, pp. 64-91, July, 2013. Article (CrossRef Link)

[22] K. Vasudeva, M. Simsek, D. Perez and I. Guvenc, "Analysis of handover failures in heterogeneous networks with fading," IEEE Transactions on Vehicular Technology, vol. 66, no. 7, pp. 6060-6074, December, 2016. Article (CrossRef Link)

[23] J. M. Moon, J. Jung, S. Lee and A. Nigam, "On the trade-off between handover failure and small cell utilization in heterogeneous networks," in Proc. of ICCW 2015: International Conference on Communication Workshop, pp.2282-2287, June 8-12, 2015. Article (CrossRef Link)

[24] Z. Xiao, T. Li, W. Ding, D. Wang and J. Zhang, "Dynamic pci allocation on avoiding handover confusion via cell status prediction in lte heterogeneous small cell networks,” Wireless Communications and Mobile Computing, vol. 16, no. 14, pp. 1972-1986, February, 2016. Article (CrossRef Link)

[25] D. Xenakis, N. Passas, L. Merakos and C. Verikoukis, "Energy-efficient and interference-aware handover decision for the LTE-Advanced femtocell network," in Proc. of ICC 2013: International Conference on Communications, pp. 2464-2468, June 9-13, 2013. Article (CrossRef Link)

[26] 3GPP TR 36.902, v9.0.0: "LTE; Evolved Universal Terrestrial Radio Access Network (E-UTRAN); Self-configuring and self-optimizing network (SON) use cases and solutions (Release 9)”, 2009. 
[27] 3GPP TR 36.839 v11.1.0: "Mobility enhancements in heterogeneous networks (release 11)”, 201

[28] X. Zhang, Z. Xiao, S. B. Mahato, and E. Liu, "Dynamic user equipment-based hysteresis-adjusting algorithm in lte femtocell networks,” IET Communications, vol. 8, no. 17, pp. 3050-3060, November, 2014. Article (CrossRef Link)

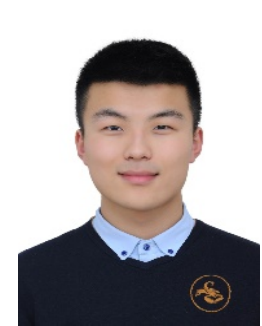

Hangyu Gu is a junior student who is pursuing the B.S. degree in communication and information system at Hunan University.

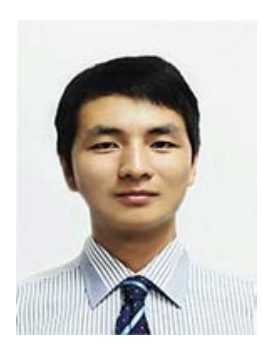

Shuangchun $\mathbf{L i}$ received the B.S. degree in information science from Hunan University, China, in 2015. He is currently pursuing the M.S. degree in communication and information system at Hunan University. His interests focus on power and resource management in future wireless and cellular networks.

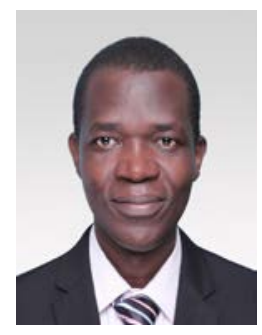

Vincent Havyarimana received his B.S. degree in Mathematics from University of Burundi, Bujumbura, in 2007. He got $\mathrm{ME}$ and $\mathrm{PhD}$ degrees in Computer Science and Technology from Hunan University, Changsha, China in 2011 and 2016 respectively. Currently, he is a Lecturer and Head of Research Service at Ecole Normale Superieure (ENS)-Burundi. He is also an academic member board of IT-Consulting and Trading Company (ICOTRACO)-Burundi. His research interests include Wireless Communication, vehicular ad-hoc networks and Mobile Computing.

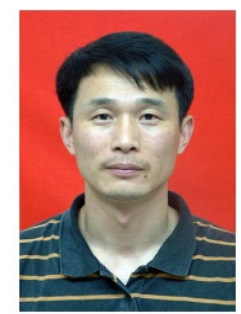

Dong Wang received the B.S. and Ph.D. degrees in computer science from Hunan University, China, in 1986 and 2006, respectively. From 2004 to 2005, he was a Visiting Scholar with the University of Technology Sydney, Australia. Since 1986, he has been with Hunan University, where he is currently a Professor. His main research interests include network test and performance evaluation, wireless communications and mobile computing.

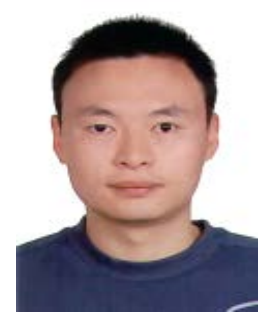

Zhu Xiao received his M.S. and Ph.D. degrees in communication and information system from Xidian University, China, in 2007 and 2010, respectively. From 2010 to 2012, he was a research fellow with the Department of Computer Science and Technology, University of Bedfordshire, U.K. He is currently an associate professor with the College of Computer Science and Electronic Engineering, Hunan University, China. He is a member of IEEE. His research interests include wireless communications and heterogeneous networks. 